\title{
Approche hiérarchisée pour le Contrôle Global du Châssis
}

\section{Hierarchical approach for Global Chassis Control}

\author{
Emna Hamrouni ${ }^{1,2,3}$, Jean-Louis Bouvin ${ }^{1,3}$, Xavier Moreau $^{1,3}$, André Benine-Neto ${ }^{1,3}$ \\ Vincent Hernette ${ }^{2,3}$, Pascal Serrier ${ }^{1,3}$ et Alain Oustaloup ${ }^{1,3}$
}

\author{
${ }^{1}$ IMS, Univ. Bordeaux, CNRS, Bordeaux INP, 351 cours de la Libération, 33405 Talence cedex, France \\ prénom.nom@ims-bordeaux.fr \\ ${ }^{2}$ Groupe PSA, 2 route de Gisy, 78943 Vélizy-Villacoublay, France \\ prénom.nom@mpsa.com \\ ${ }^{3}$ OpenLab PSA Groupe - IMS Bordeaux, 351 cours de la Libération, 33405 Talence cedex, France
}

\begin{abstract}
RÉSUMÉ. Dans un contexte de Véhicule Autonome Connecté (VAC), cet article propose une architecture hiérarchisée générique pour le Contrôle Global du Châssis (CGC). Les quatre niveaux qui composent cette architecture, à savoir : le Superviseur, la Commande Globale, la Répartition au niveau des Liaisons Au Sol (LAS) et la Commande Locale, sont détaillés. Dans une deuxième partie, un exemple dont l'objectif est d'illustrer la démarche de conception d'une telle architecture hiérarchisée est présenté. Pour des raisons didactiques, et sans que cela ne nuise à la démarche générale, le domaine d'étude de cet exemple se résume à une situation de freinage en ligne droite sur route sèche, lisse et horizontale, l'objectif étant de tenir la caisse sous sollicitations conducteur dans le domaine de fonctionnement associé au confort. Une Synthèse Fréquentielle de Commande Robuste (SFCR) fondée sur la commande CRONE est appliquée en ce qui concerne les commandes globale et locale. La comparaison des performances temporelles simulées à l'aide d'un modèle à 14 degrés de liberté du dispositif en mode actif et en mode dégradé met bien en évidence l'intérêt de l'approche proposée.
\end{abstract}

ABSTRACT. In a context of Connected Autonomous Vehicle (CAV), this paper proposes a generic hierarchical architecture for Global Chassis Control (GCC). The four levels of this architecture, namely : the Supervisor, the Global Control, the Allocation and the Local Control, are detailed. In a second part, an example whose objective is to illustrate the design process of such a hierarchical architecture is presented. For didactic reasons, and without that it doesn't harm the general approach, the field of study of this example is summed up to a braking situation in a straight line on dry, smooth and horizontal road, the objective being to hold the chassis under driving sollicitations in the operating field associated with comfort. A Robust Command Frequency Synthesis (RCFS) based on the CRONE control is applied for global and local control. The comparison of the simulated time performances using a model with 14 degrees of freedom of the device in both active and in degraded mode clearly highlights the interest of the proposed approach.

MOTS-CLÉS. Commande, régulateur robuste, dynamique du véhicule, approche hiérarchisée, suspension, CRONE, ADAS, Contrôle Global du Châssis, confort.

KEYWORDS. Control, robust controller, vehicle dynamics, hierarchical approach, suspension, CRONE, ADAS, Global Chassis Control, comfort.

\section{Introduction : contexte et problématique}

Le contexte de cet article est celui des systèmes actifs d'aide à la conduite, ou ADAS (Advanced Driver-Assistance Systems), à l'origine du développement des Véhicules autonomes et connectés (VAC) (MORAND 2014). Ces systèmes, tels que le système de freinage anti-blocage (ABS : Antilock Braking System) (BENINE-NETO 2017), le contrôle électronique de stabilité (ESC : Electronic Stability Control ou ESP : Electronic Stability Program) (MONOT 2017) ou encore le régulateur de vitesse adaptatif (ACC : Adaptive Cruise Control) (MORAND 2015) visent à améliorer, non seulement la sécurité routière, mais aussi le confort du conducteur et des passagers à l'aide du contrôle des différentes Liaisons Au Sol (LAS : Direction, Traction, Freinage et Suspension) qui agissent sur la dynamique du véhicule. Comme l'illustre la Figure 1.a, la dynamique du véhicule comporte essentiellement 14 degrés de liberté (ddl) qui se répartissent selon les trois directions : longitudinale, latérale et verticale (GILLESPIE 1992). Plus précisément, ces 14 ddl se décomposent en :

— 5 ddl pour la dynamique longitudinale : 1 translation (du véhicule) et 4 rotations (des roues); 
- 2 ddl pour la dynamique latérale : 1 translation (du véhicule) et 1 rotation (lacet);

- $7 \mathrm{ddl}$ pour la dynamique verticale : 1 translation (pompage) et 2 rotations (tangage et roulis) pour la caisse sur sa suspension et 4 translations (déplacement vertical) pour les 4 roues.

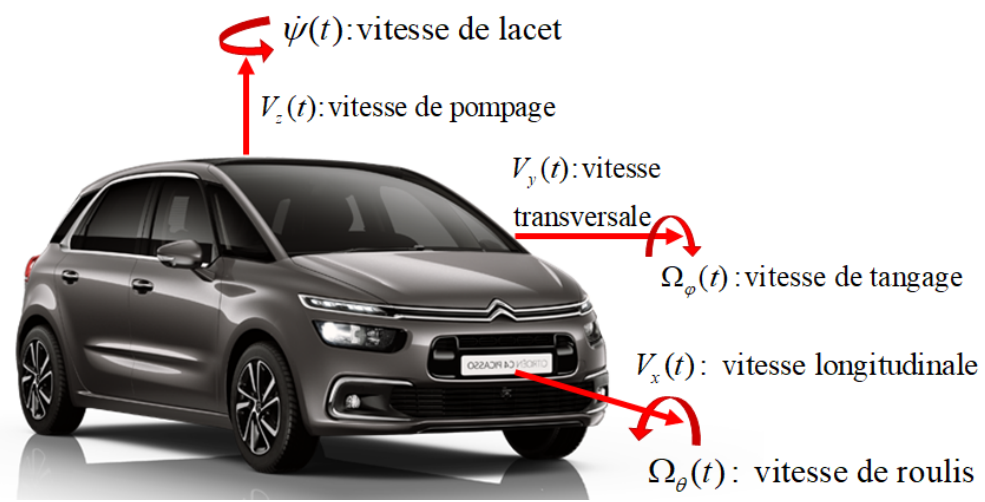

(a)

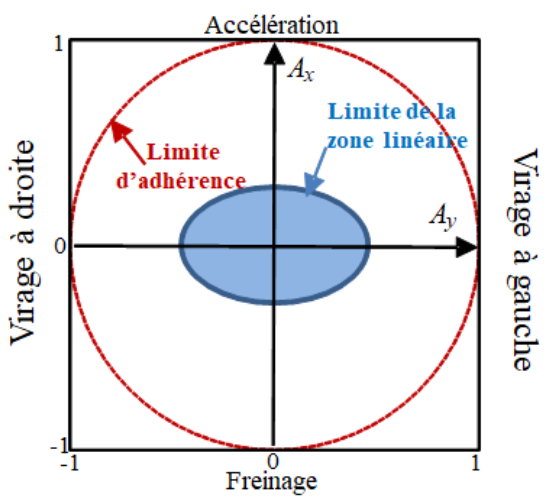

(b)

Figure 1.: Repérage des trois principales directions utilisées en dynamique du véhicule (a), diagramme des zones linéaire et non linéaire du pneumatique (b)

En raison du comportement non linéaire des pneumatiques (PACEJKA 2005), l'étude du comportement dynamique du véhicule peut être divisée en deux domaines :

- une zone linéaire correspondant aux conditions normales de conduite (elles représentent la majorité du temps d'utilisation du véhicule);

- une zone non linéaire atteinte dans des situations d'urgence (évitement d'obstacle, freinage d'urgence,... ). A titre d'illustration, la Figure 1.b présente un diagramme dans lequel l'accélération longitudinale $A_{x}$ est tracée en fonction de l'accélération latérale $A_{y}$, et ce pour une route sèche. La zone bleue représente la zone linéaire du pneumatique.

Dans un tel contexte, le pricipal problème est la coordination de l'ensemble des ADAS, encore appelé Contrôle Global du Châssis (CGC) (GAY 2000) (SENAME 2013). En matière de coordination, il existe principalement trois architectures de contrôle utilisées dans différents domaines (VIVAS 2013) (GORDON 2003) (JOHANSEN 2013)(SAE International 2014). En effet, comme l'illustre la Figure 2, on trouve :

- une architecture de contrôle décentralisé : c'est une structure parallèle à base de $N$ contrôleurs conçus et mis en œuvre de manière indépendante pour les $N$ sous-systèmes. Une communication directe entre les $N$ contrôleurs locaux est envisageable;

- une architecture de contrôle centralisé : un unique contrôleur global multivariable en charge des $N$ sous-systèmes;

- une architecture de contrôle par supervision : c'est une structure intermédiaire entre les deux précédentes. Elle est établie en ajoutant un niveau de supervision à la première, ou en ajoutant une couche intermédiaire de contrôle local à la seconde. Les contrôleurs des $N$ sous-systèmes peuvent alors être conçus et validés de manière relativement indépendante.

Après le contexte et le problème présentés dans cette introduction, la suite de cet article est organisée de la manière suivante. La section 1. propose une architecture hiérarchisée et générique pour le Contrôle Global du Châssis dont les différents niveaux y sont justifiés et détaillés. Ensuite, la section 2. traite un exemple d'illustration en commençant par définir précisément le cadre de l'étude et le scénario retenu, puis la conception de chaque niveau de l'architecture est développée. Enfin, 
les performances temporelles sont présentées mettant en évidence l'intérêt d'une telle approche. L'article conclut en rappelant les principaux points à retenir et en donnant quelques perspectives.

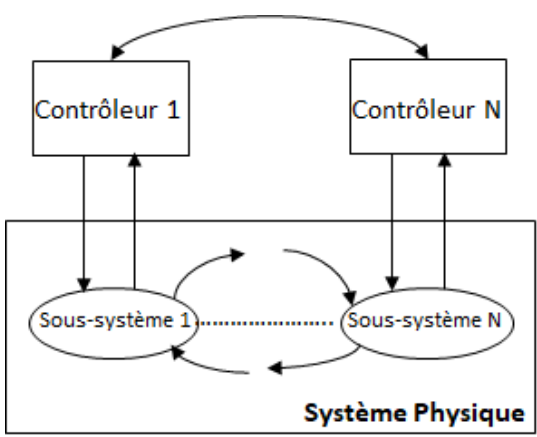

(a)

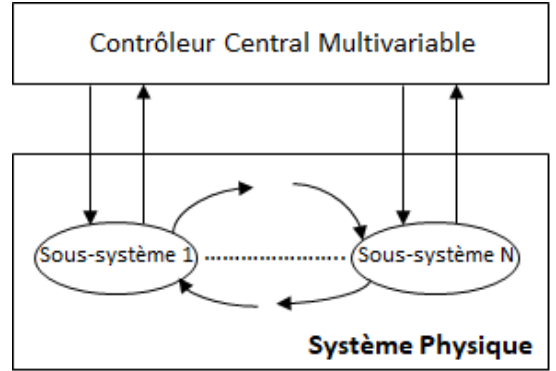

(b)

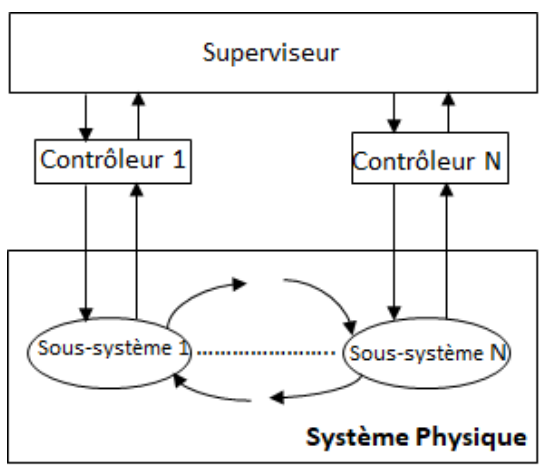

(c)

Figure 2.: Différentes architectures de contrôle : décentralisée (a), centralisée (b) et par supervision (c)

\section{Approche hiérarchisée}

Afin de faciliter la présentation de l'approche hiérarchisée développée dans cet article, des notations spécifiques sont définies. Ainsi, concernant les différentes grandeurs physiques $X(t)$ présentes dans la suite de cette étude, la convention de notation suivante est adoptée :

- $X(t)$ : variable définie dans un référentiel absolu;

- $X(t)=X^{e}+x(t)$, où $X^{e}$ est la valeur de $X(t)$ en régime stationnaire (composante statique) et $x(t)$ est la variation de $X(t)$ (composante dynamique) autour de $X^{e}$;

- $X(s)=T L\{x(t)\}$ : Transformée de Laplace de $x(t)$;

- $\tilde{X}(t)$ : Mesure de $X(t)$;

- $\hat{X}(t)$ : Estimation de $X(t)$;

- $\bar{X}(t)$ : Valeur de référence (ou désirée) de $X(t)$;

- $X_{C d G}(t)$ : Variable associée au Centre de Gravité (CdG),

Par ailleurs, les indices $\{i j\}$ sont utilisés pour repérer les quarts de véhicule $: i=1$ pour l'avant et $i=2$ pour l'arrière; $j=1$ pour la gauche et $j=2$ pour la droite.

La Figure 3 présente l'architecture proposée dans cette étude pour la commande hiérarchisée du CGC. Elle comprend quatre niveaux : le Superviseur, la Commande Globale, la Répartition au niveau des Liaisons Au Sol (LAS) et la Commande Locale. $d_{m}$ représente les entrées mesurables du conducteur (volant, pédales,...), $d$ les perturbations non mesurables (route, pente, rafale de vent, ...), $\widetilde{Y_{m}}$ l'ensemble des mesures disponibles à bord du véhicule et $\widetilde{Y_{r}}$ les mesures des sorties locales à contrôler. Les variables $U_{C G}$ et $U_{C L}$ représentent les entrées des commandes globale et locale respectivement. Cette architecture se veut générique dans le cadre du VAC. En effet, selon le niveau d'automatisation considéré parmi les cinq existants (MORAND 2014), le conducteur peut être amené à intervenir partiellement ou complètement sur les organes de conduite (volant, pédales, ... ). La suite de ce paragraphe est consacrée à la présentation des quatre niveaux composant cette architecture. 


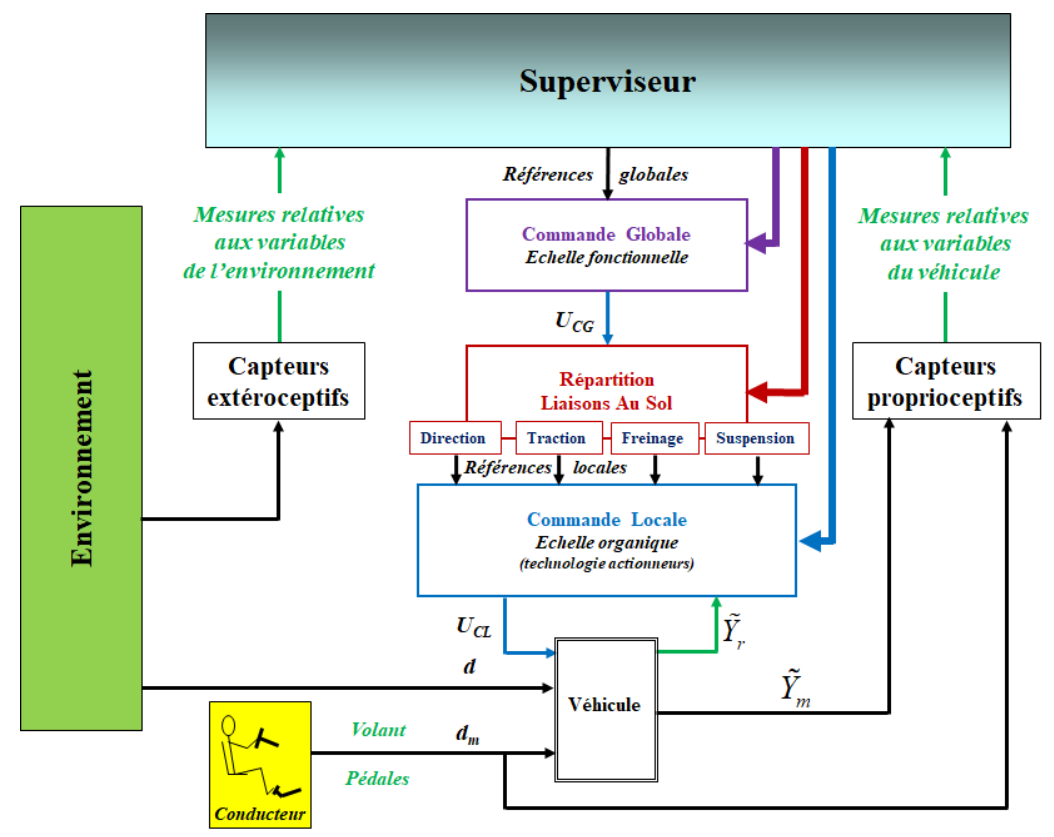

Figure 3.: Architecture proposée pour la commande hiérarchisée du CGC

\subsection{Superviseur}

Le superviseur (Figure 4.a) représente le plus haut niveau de cette architecture hiérarchisée. Il reçoit toutes les mesures disponibles en provenance, non seulement du véhicule (capteurs proprioceptifs : centrale inertie,...), mais aussi de l'environnement (capteurs extéroceptifs : caméra, scanner-laser,...), ou encore des informations communiquées par l'infrastructure routière ou par d'autres véhicules (partie connectée du véhicule autonome). Toutes ces données sont utilisées dans les modules Perception de l'environnement et Observation des grandeurs véhicules, modules en relation avec les modules Planification de trajectoire et Génération de mouvement, ainsi que Diagnostic.

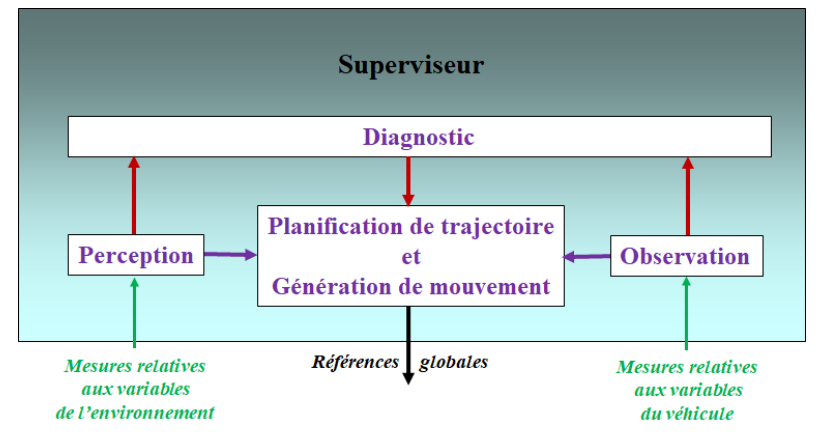

(a)

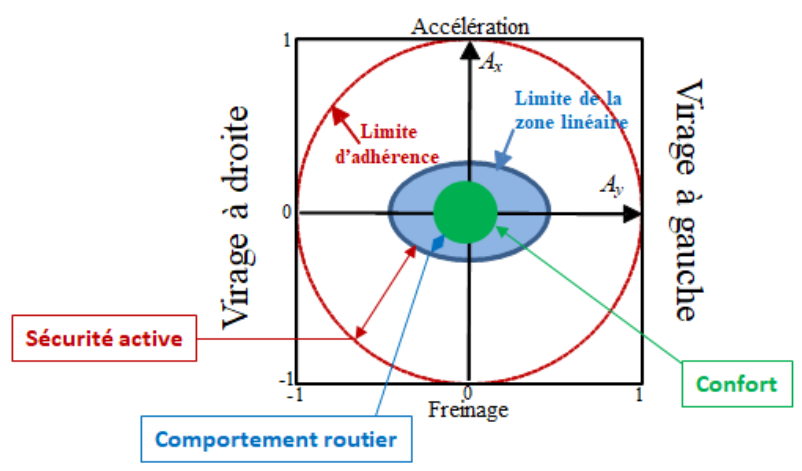

(b)

Figure 4.: Modules au sein du Superviseur (a) et illustration dans un diagramme $A_{x}=\mathrm{f}\left(A_{y}\right)$ des trois domaines de fonctionnement : confort (zone verte), comportement routier (zone bleue) et sécurité active (zone non linéaire du pneumatique) (b)

A partir de ces informations, le Superviseur estime le domaine de fonctionnement dans lequel se trouve le véhicule, à savoir Confort, Comportement routier ou Sécurité active. La Figure 4.b illustre dans un diagramme $A_{x}=\mathrm{f}\left(A_{y}\right)$, sur route sèche, ces trois domaines de fonctionnement. 
Le premier domaine de fonctionnement est défini par des accélérations longitudinale et latérale inférieures à environ $0.3 \mathrm{~g}$ en valeur absolue. Dans cette zone, la dynamique verticale du véhicule, à travers le système de suspension, n'a pas d'influence significative sur la dynamique horizontale (longitudinale et latérale) (LETEVE 2014). Ainsi, le confort vibratoire peut être maximisé à travers l'isolation vibratoire et la tenue de caisse sous sollicitations route et conducteur. Dans le second domaine de fonctionnement (zone bleue), le système de suspension commence à avoir une influence sur la dynamique horizontale, de sorte que le dilemme confort/comportement routier doit être géré en utilisant une stratégie de contrôle adaptée et différente de celle orientée confort. Enfin, dans la zone non linéaire du pneumatique, la sécurité est prioritaire. L'objectif est de maximiser la tenue de route du véhicule à l'aide des moyens actifs des LAS, à savoir les systèmes : de suspension (pour la tenue de roue), de direction (pour le microbraquage) et de freinage (pour le microfreinage). A noter que la présence de moteurs électriques de traction dans les roues offre un moyen d'action supplémentaire dans le contrôle de la tenue de route. Ainsi, les valeurs des indicateurs pertinents de la dynamique du véhicule doivent être actualisées en temps réel, et ce afin de permettre au superviseur de reconnaître le domaine de fonctionnement et de choisir la stratégie de commande adaptée.

\subsection{Commande Globale}

La Commande Globale (CG) exécute la stratégie de contrôle associée au domaine de fonctionnement estimé par le superviseur (Figure 3). Elle est conçue pour générer les forces et les couples de référence appliqués au $\mathrm{CdG}$ du véhicule conformément à la planification de trajectoire et la génération de mouvement définies au niveau du superviseur. Les variables de haut niveau à contrôler sont donc les vitesses de translation et de rotation associées aux ddl du CdG (Figure 1.a). Par exemple, dans une situation de conduite correspondant à la zone de confort, l'objectif est de maîtriser la tenue de caisse, non seulement dans le cadre des sollicitations du conducteur (reports de charge), mais également dans le cadre des sollicitations de la route. Cet objectif se résume à :

$$
\left\{\begin{array}{l}
\text { - vitesse de Pompage }: V_{z}(t)=0 \\
\text { - vitesse de Tangage }: \Omega_{\varphi}(t)=0 \\
\text { - vitesse de Roulis }: \Omega_{\theta}(t)=0
\end{array},\right.
$$

encore appelé PTR = 0 (Pompage, Tangage, Roulis =0), (GAY 2000).

Un autre exemple de commande globale est présenté dans (MONOT 2017) pour le contrôle de la stabilité d'un véhicule à travers la régulation de la vitesse de lacet.

\subsection{Répartition}

Le troisième niveau de cette architecture hiérarchisée correspond à la répartition des forces et des couples de référence au niveau des LAS. Il joue un rôle essentiel pour le passage de la commande globale (définie à l'échelle fonctionnelle indépendamment de la technologie) à la commande locale (définie à l'échelle organique au niveau des actionneurs) (JOHANSEN 2013). Par exemple dans le cadre de l'objectif $\mathrm{PTR}=0$ (relation $(1)$ ), ce niveau assure la répartition des efforts de référence aux quatre suspensions (GAY 2000). Plus généralement, connaissant le nombre $N_{o}$ d'objectifs formulés analytiquement et le nombre $N_{a}$ d'actionneurs disponibles, le problème de répartition se résume à trois cas (ATTIA 2014) : 
- Si $N_{a}=N_{o}$ alors il existe une relation de passage des commandes globales aux commandes locales, et réciproquement;

- Si $N_{a}>N_{o}$ alors le système est sur-actionné; les actionneurs ayant le plus grand potentiel sont choisis afin de revenir au premier cas;

- Si $N_{a}<N_{o}$ alors le système est sous-actionné; il est alors possible de réduire le nombre d'objectifs afin de revenir au premier cas, ou bien de reformuler le problème en un problème d'optimisation où les signaux de la Commande Locale sont calculés en minimisant une fonction objectif soumise à des contraintes liées aux limites physiques des actionneurs.

\subsection{Commande Locale}

Enfin, le quatrième niveau est celui de la Commande Locale (CL) pour laquelle les références sont basées sur les niveaux précédents (CG et répartition). Ainsi, la commande locale des actionneurs des LAS permet de contrôler les variables de bas niveau, à savoir :

- l'angle de braquage des roues directrices (suivi de trajectoire, contrôle de stabilité par microbraquage, etc);

- la vitesse de rotation des roues pour les cas de traction (ACC, ASR, etc) et de freinage (ESC, ABS, etc) (BENINE-NETO 2017) et (NOUILLANT 2002);

- les déplacements verticaux des roues (masses non suspendues) et des quatre coins du châssis (masse suspendue) (BOUVIN 2017).

\section{Exemple d'illustration}

Ce paragraphe présente un exemple dont l'objectif est d'illustrer la démarche de conception d'une telle architecture hiérarchisée. Volontairement pour des raisons didactiques, et sans que cela ne nuise à la démarche générale, le domaine d'étude de cet exemple se résume à une situation de freinage en ligne droite sur route sèche, lisse et horizontale. Dans une première phase du scénario, le véhicule se déplace à vitesse longitudinale $V_{x}$ constante. Dans une deuxième phase, le système de freinage est activé à l'aide d'une rampe tronquée dont la durée et la pente sont calibrées pour que la décélération longitudinale $A_{x}(t)$ soit inférieure en valeur absolue à $0.3 \mathrm{~g}$ de manière à ce que le domaine de fonctionnement reste dans la zone confort (zone verte (Figure 4.b)). Cette phase de freinage génère un couple de tangage $C_{t 0}(t)$ dont l'expression est donnée par (GILLESPIE 1992) :

$$
C_{t 0}(t)=-H M_{T} A_{x}(t),
$$

où $H$ représente la hauteur du $\mathrm{CdG}$ et $M_{T}$ la masse totale du véhicule. Si on suppose que le châssis est indéformable, alors les reports de charge $F_{0} \_i j(t)$ vus par les quatre quarts du véhicule et résultant du couple $C_{t 0}(t)$ de mise en roulis sont donnés par (GILLESPIE 1992) :

$$
\left\{\begin{array}{l}
F_{0 \_11}(t)=F_{0 \_12}(t)=+\frac{1}{2 L} C_{t 0}(t) \\
F_{0 \_21}(t)=F_{0 \_22}(t)=-\frac{1}{2 L} C_{t 0}(t)
\end{array},\right.
$$

où $L$ représente l'empattement du véhicule (distance entre les deux essieux). Dans le cas d'une suspension passive, ces reports de charge longitudinaux entraînent la plongée du véhicule (mouvement de tangage). A noter qu'en dehors d'un freinage d'urgence (GILLESPIE 1992), la décélération 
longitudinale $A_{x}(t)$ est proportionnelle au déplacement $X_{p}(t)$ de la pédale de frein, soit :

$$
A_{x}(t)=\lambda_{x} X_{p}(t)
$$

$\lambda_{x}$ étant une constante fonction du système de freinage et des paramètres inertiels et géométriques du véhicule considéré. Dans cette étude, le véhicule considéré est un C4 Picasso (Figure 1.a) équipé d'un régulateur d'assiette permettant de tenir la caisse sous sollicitations conducteur lors des phases de freinage et de mise en virage. Afin de limiter volontairement la consommation énergétique, le domaine de fonctionnement de ce régulateur d'assiette est limité à la zone confort (Figure 4.b).

Par ailleurs, modèle à 14 ddl de la dynamique du véhicule (non présenté dans cet article) développé sous MatLab/Simulink est utilisé pour les simulations temporelles de la dynamique du véhicule, en particulier lors de la phase finale de validation des lois de commande (modèle de validation) (LETEVE 2014).

Les sections suivantes présentent, de manière plus détaillée, chacun des quatre niveaux composant la commande hiérarchisée du CGC qui, dans le cas particulier de l'exemple d'illustration, se limite au Contrôle Global de la Suspension (CGS).

\subsection{Superviseur}

Comme l'illustre la Figure 4, le superviseur reçoit, entre autres, les mesures :

— des déplacements verticaux $\tilde{Z}_{2 \_i j}(t)$ et des vitesses verticales $\tilde{V}_{2 \_i j}(t)$ des quatre quarts du véhicule;

- du déplacement $\tilde{X}_{p}(t)$ de la pédale de frein;

- de la vitesse de tangage $\tilde{\Omega}_{\varphi}(t)$ du CdG.

A partir de ces mesures, le couple de mise en tangage $\hat{C}_{t 0}(t)$ est estimé à l'aide d'une relation de la forme :

$$
\hat{C}_{t 0}(t)=H_{0} M_{T 0} \lambda_{x} \tilde{X}_{p}(t)
$$

où $H_{0}$ représente la hauteur du CdG associée à la masse totale à vide $M_{T 0}$.

Dans cette étude, la masse totale $M_{T}$ du véhicule est considérée comme un paramètre incertain ne faisant pas l'objet d'une estimation. Ainsi, la seule valeur supposée connue est sa valeur à vide $M_{T 0}$ inscrite sur la carte grise du véhicule. De la même manière, les forces $\hat{F}_{s p \_i j}(t)$ développées par les éléments passifs des quatre suspensions sont estimées à l'aide d'une relation de la forme :

$$
\hat{F}_{s p \_i j}(t)=k_{2 \_i j} \tilde{Z}_{2 \_i j}(t)+b_{2 \_i j} \tilde{V}_{2 \_i j}(t)
$$

où $k_{2 \_i j}$ et $b_{2 \_i j}$ représentent les valeurs fournies par le constructeur de la raideur du ressort et du coefficient de frottement visqueux de l'amortisseur de la suspension $\{i j\}$ respectivement. Pour le scénario retenu, la Figure 5 présente le déplacement $X_{p}(t)$ de la pédale de frein généré par le conducteur (Figure 5.a), ainsi que la décélération $A_{x}(t)$ (Figure 5.b) et le couple de mise en tangage $C_{t 0}(t)$ (Figure 5.c) qui en résultent. Ces tracés sont obtenus à l'aide du simulateur à 14 ddl et permettent de bien vérifier :

- la cohérence entre la définition du domaine d'étude (fonctionnement dans la zone confort de la Figure 4.b) et le calibrage de la sollicitation conducteur (déplacement pédale de frein);

- le caractère linéaire entre le couple de mise en roulis $C_{t 0}(t)$, la décélération longitudinale $A_{x}(t)$ et le déplacement $X_{p}(t)$ de la pédale de frein. 


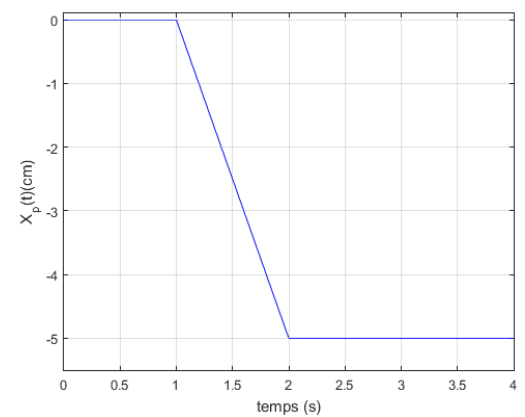

(a)

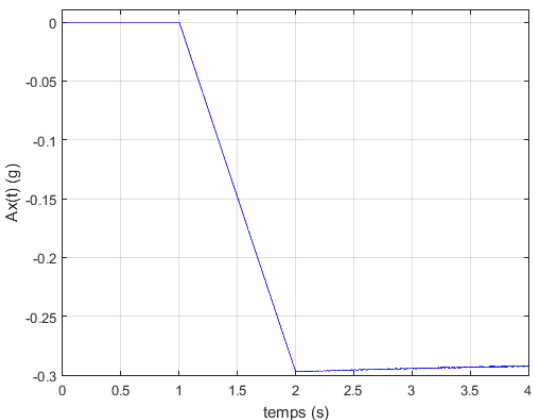

(b)

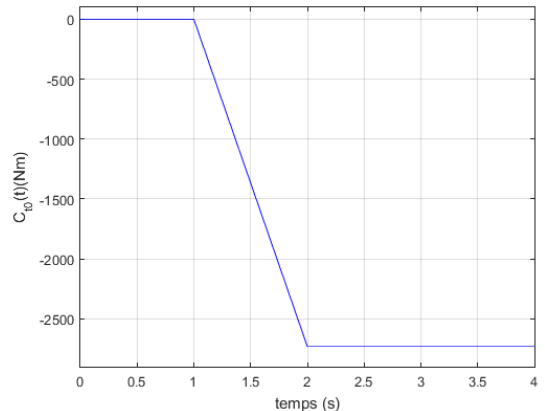

(c)

Figure 5.: Déplacement $X_{p}(t)$ de la pédale de frein généré par le conducteur (a), décélération $A_{x}(t)$ (b) et couple de mise en tangage $C_{t 0}(t)$ pour le scénario retenu

\subsection{Commande Globale}

L'objectif de la commande globale est la régulation de la vitesse de tangage $\Omega_{\varphi}(t)$ autour de zéro lors des phases de freinage se situant dans la zone de confort. La méthodologie utilisée pour la conception de la commande globale est une Synthèse Fréquentielle de Commande Robuste (SFCR), et plus précisément la commande CRONE (LANUSSE 2013). Le modèle de synthèse est défini à partir du Principe Fondamental de la Dynamique (PFD) appliqué à l'inertie de tangage $I_{y y}$ du véhicule et écrit sous sa forme causale intégrale, soit :

$$
\Omega_{\varphi}(t)=\frac{1}{I_{y y}} \int_{0}^{t} C_{\Sigma y}(\tau) d \tau+\Omega_{\varphi}(0),
$$

où $C_{\Sigma_{y}}(t)$ est le couple résultant égal à la somme algébrique des couples extérieurs appliqués à l'inertie de tangage $I_{y y}$ soit :

$$
C_{\Sigma_{y}}(t)=C_{t 0}(t)+C_{a t}(t)
$$

Le couple anti-tangage de référence (ou désiré), noté $\bar{C}_{a t}(t)$, résulte de la somme d'un couple d'anticipation $C_{F F}(t)$ issu d'un bloc FeedForward et d'un couple $C_{F B}(t)$ issu d'un régulateur, soit :

$$
\begin{aligned}
& \bar{C}_{a t}(t)=C_{F F}(t)+C_{F B}(t), \\
& \text { où } C_{F F}(t)=-\hat{C}_{t 0}(t) \text { et } C_{F B}(s)=-K_{G} \tilde{\Omega}_{\varphi}(s) .
\end{aligned}
$$

La Figure 6 présente le schéma de synthèse utilisé pour le calcul du régulateur $K_{G}(s)$ où la vitesse de tangage $\Omega_{\varphi}(s)$ est obtenue à partir de la transformée de Laplace de l'équation (7) sous l'hypothèse de conditions initiales nulles. A noter que cette hypothèse est cohérente avec la phase initiale du scénario où le véhicule se déplace à vitesse longitudinale $V_{x}$ constante et donc où $\Omega_{\varphi}(0)=0$. La fonction de transfert $P(s)$ qui apparait alors entre $C_{\Sigma y}(s)$ et $\Omega_{\varphi}(s)$ est un intégrateur avec un paramètre inertiel $I_{y y}$ dont la valeur varie entre la configuration à vide et celle totale en charge du véhicule, soit :

$$
P(s)=\frac{1}{I_{y y} s},
$$




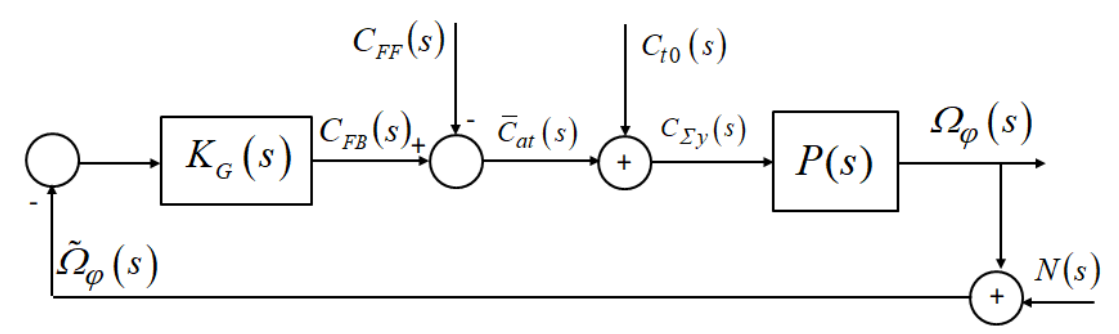

Figure 6.: Schéma de synthèse utilisé pour le calcul du régulateur $K_{G}(s)$

où le paramètre incertain $I_{y y} \in[2615 ; 3740] \mathrm{kg} \cdot \mathrm{m}^{2}$.

Par ailleurs, la traduction des spécifications du cahier des charges en matière de commande conduit à :

— pour le degré de stabilité : un facteur de résonance de la fonction de sensibilité complémentaire $Q_{T} \leqslant 3 \mathrm{~dB}$;

- pour la rapidité : une bande passante la plus grande possible, sachant que l'on souhaite une bande passante minimale au moins quatre fois plus rapide que la bande passante $\mathrm{du}$ conducteur dont la borne supérieure est estimée au voisinage de $2.5 \mathrm{~Hz}$;

— pour la précision en régime permanent : une erreur relative inférieure à $1 \%$;

- pour la limite de la commande $: \max \left|\overline{C_{a t}}\right| \leqslant 16000 \mathrm{Nm}$;

A partir des données de synthèse (constituées par l'ensemble des spécifications du cahier des charges, du transfert $P(s)$, des incertitudes paramétriques et de la définition des signaux d'entrée pour le domaine d'étude retenu), un régulateur CRONE de deuxième génération est synthétisé. Pour des raisons de place, les différentes étapes de la synthèse, comme par exemple celle de la mise en forme de la boucle (loop shaping) ou encore le passage de la forme fractionnaire à la forme rationnelle du régulateur, ne peuvent être détaillées dans cet article. Le lecteur intéressé trouvera dans la littérature de nombreuses références bibliographiques sur le sujet comme par exemple (OUSTALOUP 1991) (OUSTALOUP 2000) (LANUSSE 2013) et bien d'autres encore... Ainsi, conformément à la démarche de SFCR développée pour la commande CRONE de deuxième génération, l'expression fractionnaire de la fonction de transfert $K_{G}(s)$ du régulateur de la commande globale obtenue est de la forme :

$$
K_{G}(s)=\beta_{0} I_{y y 0} s\left(\frac{1+s / \omega_{l}}{s / \omega_{l}}\right)^{n_{l}}\left(\frac{1+s / \omega_{h}}{1+s / \omega_{l}}\right)^{n_{u}} \frac{1}{\left(1+s / \omega_{h}\right)^{n_{h}}},
$$

où $\beta_{0}$ est une constante positive, $I_{y y 0}$ l'inertie de tangage à vide, $\omega_{l}$ et $\omega_{h}$ des fréquences transitionnelles telles que $\omega_{l}<\omega_{h}, n_{l}$ l'ordre aux basses fréquences de la fonction de transfert en boucle ouverte, notée $\beta_{G}(s), n_{u}$ l'ordre de $\beta_{G}(s)$ au voisinage de la fréquence au gain unité, notée $\omega_{u}$, et $n_{h}$ l'ordre de $\beta_{G}(s)$ aux hautes fréquences. En prenant $n_{l}=n_{h}=2$ (comportement double intégrateur de $\beta_{G}(s)$ aux basses et hautes fréquences) et $n_{u}=1.5$ (comportement intégrateur d'ordre non entier 1.5 de $\beta_{G}(s)$ pour tangenter le contour d'amplitude $Q_{T}=3 d B$ dans le plan de Nichols), l'expression (12) de $K_{G}(s)$ se résume à :

$$
K_{G}(s)=\frac{K_{0}}{s}\left(\frac{1+s / \omega_{l}}{1+s / \omega_{h}}\right)^{m}
$$

où $K_{0}=\beta_{0} I_{y y 0} \omega_{l}^{2}$ et $m=2-n_{u}$. Finalement, les valeurs numériques des quatre paramètres de la forme fractionnaire du régulateur CRONE sont : $m=0.5, \omega_{l}=7.15 \mathrm{rad} / \mathrm{s}, \omega_{h}=551 \mathrm{rad} / \mathrm{s}$ et $K_{0}=2.6910^{6} \mathrm{Nms} / \mathrm{rad}$. 
Toujours pour des raisons de place, seules les réponses fréquentielles de la fonction de transfert en boucle ouverte $\beta_{G}(s)$ sont présentées dans les plans de Bode (Figure 7.a) et Nichols (Figure 7.b) pour l'inertie minimale (en bleu) et l'inertie maximale (en rouge). Conformément aux objectifs de la commande CRONE (OUSTALOUP 1991), la Figure 7.b met bien en évidence la robustesse du degré de stabilité vis-à-vis des variations de l'inertie (d'un facteur 1.43) avec un gabarit vertical qui tangente le même contour d'amplitude à $3 \mathrm{~dB}$ quelle que soit la valeur de $I_{y y}$. Quant à la rapidité de la commande, fixée par la valeur de $\omega_{u}$, elle diminue quand l'inertie augmente, passant ainsi d'une valeur de $\omega_{u}=62.8 \mathrm{rad} / \mathrm{s}$ à vide à $\omega_{u}=49.3 \mathrm{rad} / \mathrm{s}$ en charge.

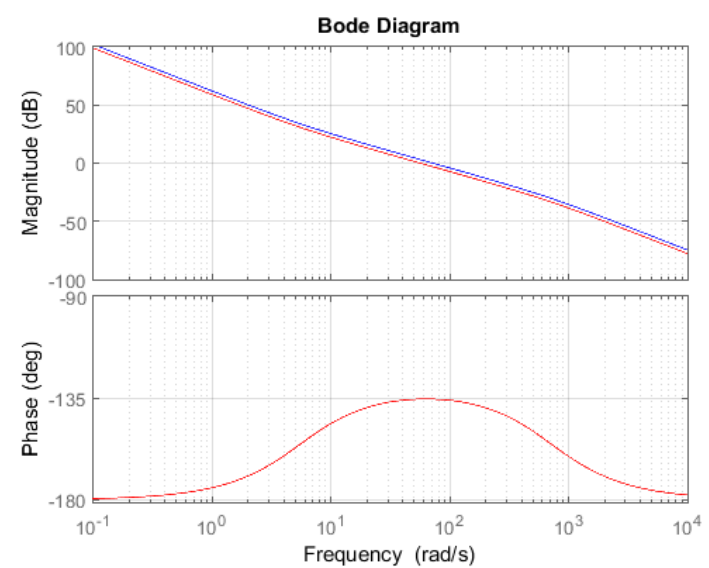

(a)

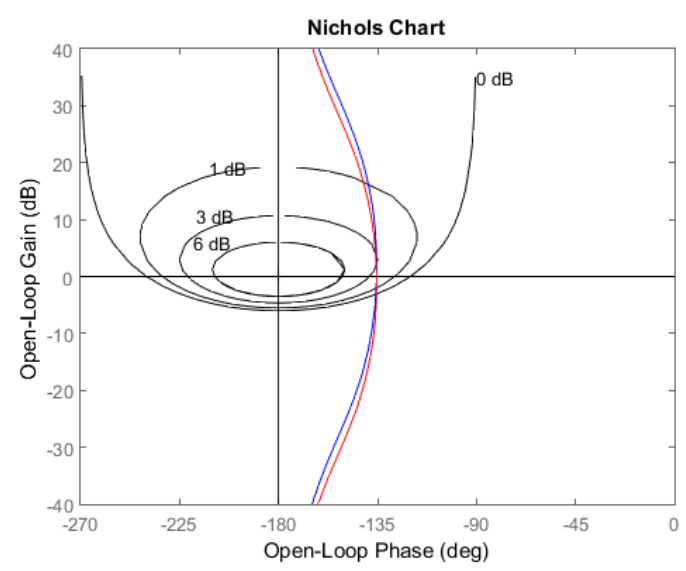

(b)

Figure 7.: Diagrammes de Bode (a) et de Nichols (b) de la fonction de transfert en boucle ouverte $\beta_{G}(s)$ pour l'inertie minimale (bleu) et l'inertie maximale (rouge)

\subsection{Répartition}

Le rôle du niveau Répartition est d'assurer le passage du couple anti-tangage de référence $\bar{C}_{a t}(t)$ aux efforts $\bar{F}_{s \_i j}(t)$ que doivent développer les quatre suspensions pour atteindre l'objectif de tenir la caisse sous sollicitation conducteur. Idéalement, cet objectif est atteint si les efforts $F_{s \_i j}(t)$ générés par les quatre suspensions sont égaux et opposés aux reports de charge $F_{0 \_i j}(t)$. Par conséquent, si on suppose que le châssis est indéformable, les valeurs de référence $\bar{F}_{s \_i j}(t)$ sont déterminées à partir de $\bar{C}_{a t}(t)$ conformément à la relation :

$$
\left\{\begin{array}{l}
\bar{F}_{s \_11}(t)=\bar{F}_{s \_12}(t)=+\frac{1}{2 L} \bar{C}_{a t}(t) \\
\bar{F}_{s \_21}(t)=\bar{F}_{s \_22}(t)=-\frac{1}{2 L} \bar{C}_{a t}(t)
\end{array} .\right.
$$

\subsection{Commande Locale}

Quatre boucles de commande locale sont réalisées à ce dernier niveau pour assurer la régulation autour de zéro des variations des déplacements verticaux $Z_{2 \_i j}(t)$ des masses suspendues des quatre quarts du véhicule. La Figure 8.a présente un modèle quart de véhicule à 2 ddl où $Z_{0 \_i j}(t)$ représente l'élongation verticale du profil routier, $Z_{1 \_i j}(t)$ les déplacements verticaux des masses non suspendues (roues), $m_{1 \_i j}$ et $m_{2 \_i j}$ étant, respectivement, les masses non suspendues et suspendues, $k_{1 \_i j}$ et $b_{1 \_i j}$ désignant, respectivement, la rigidité verticale et le coefficient de frottement 
visqueux équivalent du pneumatique. Compte tenu du domaine d'étude, à savoir un déplacement sur une route horizontale et lisse (pas d'entrée route : $Z_{0 \_i j}(t)=0$ ) et de la très faible sensibilité du déplacement vertical $Z_{1 \_i j}(t)$ des masses suspendues aux reports de charge dans la zone confort (LETEVE 2014), un modèle quart de véhicule à 1 ddl est retenu pour la synthèse des régulateurs locaux où les paramètres incertains sont les masses suspendues $m_{2 \_i j}$ variant entre à vide et en charge. Ainsi, chaque suspension développe une force $F_{s_{-} i j}(t)$ qui résulte de la somme de deux

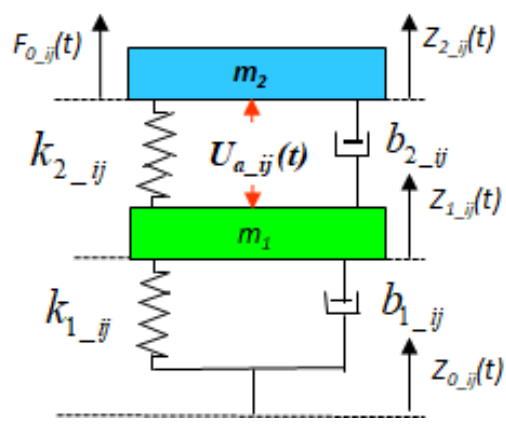

(a)

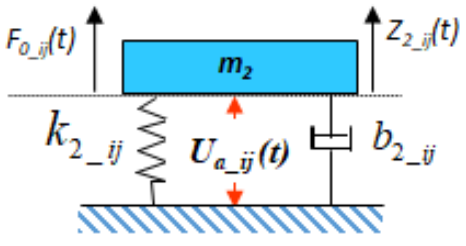

(b)

Figure 8.: Modèle quart de véhicule : pour la validation (a) et pour la synthèse (b)

composantes : $F_{s p \_i j}(t)$ développée par les éléments passifs (ressort et amortisseur) et $U_{a \_i j}(t)$ développée par l'actionneur, soit :

$$
F_{s \_i j}(t)=F_{s p \_i j}(t)+U_{a \_i j}(t) .
$$

La force $U_{a \_i j}(t)$ développée par chaque actionneur résulte elle-même de la somme d'un terme d'anticipation (FeedForward) $U_{F F \_i j}(t)$ et d'un terme de régulation (FeedBack) $U_{F B \_i j}(t)$, soit :

$$
U_{a \_i j}(t)=U_{F F \_i j}(t)+U_{F B \_i j}(t),
$$

où la force $U_{F F \_i j}(t)$ est définie par la différence entre la force de référence $\bar{F}_{s \_i j}(t)$ que doit développer la suspension et la force estimée $\hat{F}_{s p \_i j}(t)$ développée par les éléments passifs, soit :

$$
U_{F F \_i j}(t)=\bar{F}_{s \_i j}(t)-\hat{F}_{s p \_i j}(t) .
$$

De plus, la fonction de transfert $G(s)$ du modèle utilisé pour la synthèse (Figure 8.b) entre la commande en force $U_{a \_i j}(s)$ et le déplacement vertical $Z_{2 \_i j}(s)$ est donnée par :

$$
G(s)=\frac{Z_{2 \_i j}(s)}{U_{a \_i j}(s)}=\frac{1}{m_{2 \_i j} s^{2}+b_{2 \_i j} s+k_{2 \_i j}},
$$

où, $m_{2 \_i j} \in[270.7 ; 513.2] \mathrm{kg}, k_{2 \_i j}=9644.8 \mathrm{~N} / \mathrm{m}$ et $b_{2 \_i j}=1620 \mathrm{Ns} / \mathrm{m}$. La Figure 9 présente le schéma fonctionnel utilisé pour la synthèse de la commande locale.

Pour des raisons de place disponible, et sans que cela n'affecte le caractère générique de la démarche, les spécifications du cahier des charges pour la commande locale sont choisies identiques à celles de la commande globale, seule la spécification sur le maximum est différente puisqu'il s'agit ici d'une commande en force (et non en couple) limitée à $4000 \mathrm{~N}$ par actionneur. Ainsi, à partir des données de synthèse, un régulateur CRONE de première génération est synthétisé. Toujours pour des raisons de place, les différentes étapes de la synthèse ne sont pas détaillées dans cet 


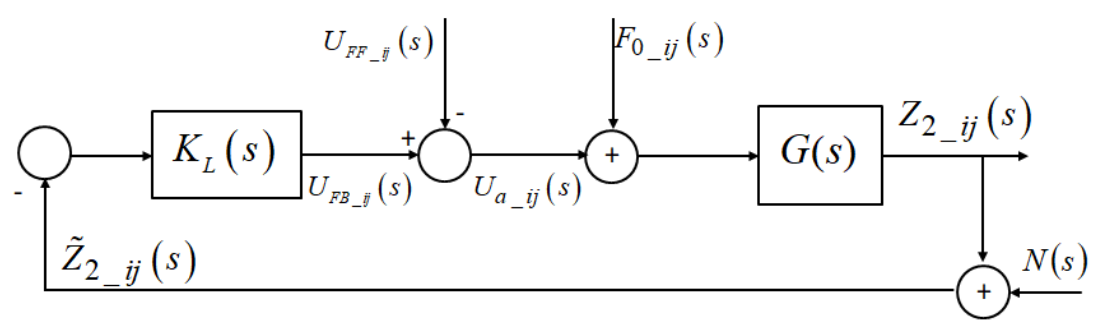

Figure 9.: Schéma fonctionnel pour la synthèse de la commande locale

article. A noter qu'un seul et unique régulateur CRONE identique pour les quatre suspensions est synthétisé. Ainsi, conformément à la démarche de SFCR développée pour la commande CRONE de première génération, l'expression fractionnaire de la fonction de transfert $K_{L}(s)$ du régulateur de la commande locale obtenue est de la forme :

$$
K_{L}(s)=C_{0}\left(\frac{1+s / \omega_{1}}{s / \omega_{1}}\right)^{m_{l}}\left(\frac{1+s / \omega_{2}}{1+s / \omega_{3}}\right)^{m}\left(\frac{1}{1+s / \omega_{4}}\right)^{m_{h}},
$$

où $m_{l}=2, m=0.5, m_{h}=1, \omega_{1}=\omega_{2}=4.1 \mathrm{rad} / \mathrm{s}$ et $\omega_{3}=\omega_{4}=628.3 \mathrm{rad} / \mathrm{s}, C_{0}=$ $2.7210^{5} \mathrm{Ns} / \mathrm{rad}$.

Comme pour la commande globale, seules les réponses fréquentielles de la fonction de transfert en boucle ouverte $\beta_{L}(s)$ sont présentées dans les plans de Bode (Figure 10.a) et Nichols (Figure 10.b) pour les masses suspendues minimale (en bleu) et maximale (en rouge). Là encore, la Figure 10.b met bien en évidence la robustesse du degré de stabilité vis-à-vis des variations de la masse suspendue (d'un facteur 1.89) avec un gabarit vertical qui tangente le même contour d'amplitude de $3 \mathrm{~dB}$ quelle que soit la valeur de $m_{2 \_i j}$. Quant à la rapidité de la commande, fixée par la valeur de $\omega_{u}$, elle diminue quand la masse augmente, passant ainsi d'une valeur de $\omega_{u}=62.8 \mathrm{rad} / \mathrm{s}$ à vide à $\omega_{u}=40.1 \mathrm{rad} / \mathrm{s}$ en charge.
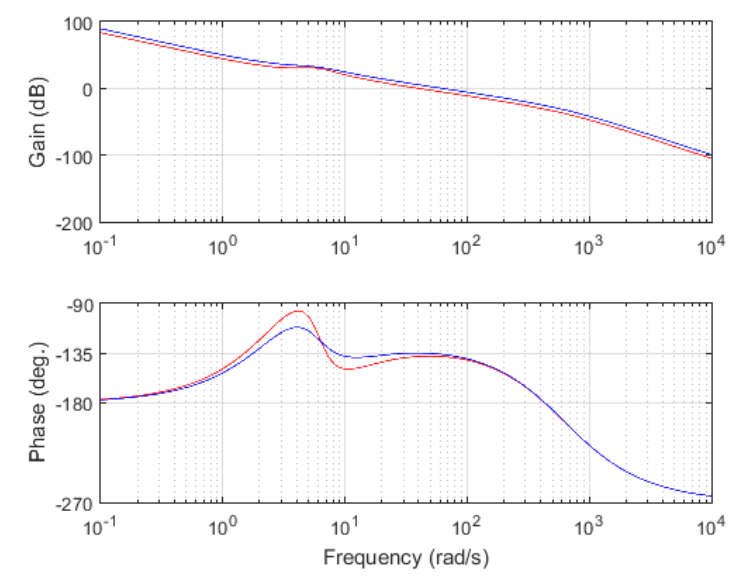

(a)

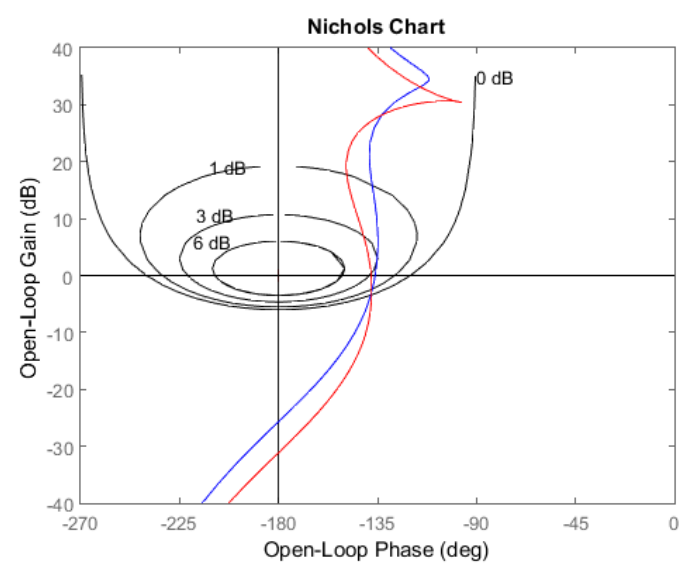

(b)

Figure 10.: Diagrammes de Bode (a) et de Nichols (b) de la fonction de transfert en boucle ouverte $\beta_{L}(s)$ pour la masse minimale (bleu) et la masse maximale (rouge) 


\section{Analyse des résultats}

A l'aide du modèle à 14 ddl, pour le scénario retenu et dans le cadre de la commande hiérarchisée telle que définie dans cette étude, la Figure 11.a présente les tracés du Jerk de tangage $J_{\varphi}(t)$ (dérivée première de l'accélération par rapport au temps) obtenus à vide (en bleu) et en charge (en rouge) avec le système en mode actif. Les niveaux de Jerk obtenus sont très faibles dans le sens où ils sont acceptables par le corps humain et ne produisent pas de l'inconfort. La Figure 11.b présente, avec la même légende les tracés de l'accélération de tangage $A_{\varphi}(t)$ obtenus avec le système en mode actif (en trait continu) et en mode dégradé (en traits pointillés), c'est-à-dire avec les quatre actionneurs en panne. En mode actif. L'accélération de tangae est très proche de zéro, à un niveau non perceptible par les occupants du véhicule. La Figure 11.c présente, toujours avec la même légende, les tracés de la vitesse de tangage $\Omega_{\varphi}(t)$. Là aussi, en mode actif, la vitesse de tangage est très proche de zéro, à un niveau non perceptible par les occupants du véhicule. La Figure 11.d présente, avec la même légende, les tracés de l'angle de tangage $\varphi(t)$ résultant de l'intégration de la vitesse $\Omega_{\varphi}(t)$. De la même façon, en mode actif, l'angle de tangage est très proche de zéro, à un niveau non perceptible par les occupants du véhicule. Les tracés de ces quatre variables de haut niveau, c'est-à-dire situées au niveau du CdG, illustrent bien la tenue de caisse sous sollicitation conducteur. Un zoom a été fait sur les tracés de l'accélération $A_{\varphi}(t)$, de la vitesse $\Omega_{\varphi}(t)$ ainsi que de l'angale $\varphi(t)$ de tangage(figures 11.b, c et d) afin d'observer le faible niveau de chacune de ces variables en mode actif. Les figures 11.e et $\mathrm{f}$ illustrent les résultats au niveau organique. Ainsi, la Figure 11.e présente les reports de charge $F_{0 \_i j}(t)$ appliqués aux quatre coins du véhicule à vide (en bleu) et en charge (en rouge) ainsi que les efforts $U_{a \_i j}(t)$ (somme des composantes FeedForward et FeedBack) développés par les quatre actionneurs. Cette figure illustre, notamment, le fait que les efforts des actionneurs sont bien de signe opposé et d'amplitude très proche de ceux des reports de charge. Afin de quantifier la contribution de chacune de ces deux composantes FeedForward $U_{F F \_i j}(t)$ et FeedBack $U_{F B \_i j}(t)$, la figures 11.f présente leurs tracés. Pour rappel, dans cette étude, la masse totale $M_{T}$ du véhicule est considérée comme un paramètre incertain ne faisant pas l'objet d'une estimation. La seule valeur supposée connue au niveau du superviseur, et utilisée pour l'anticipation, est sa valeur à vide $M_{T 0}$. Ainsi, lorsque le véhicule est à vide, d'une part, et en l'absence (cas idéal) de bruit de mesure et de perturbation autre que les reports de charge, d'autre part, la composante FeedForward $U_{F F \_i j}(t)$ (tracé en bleu Figure 11.f) représente $100 \%$ de l'effort $U_{a \_i j}(t)$ développé par chaque actionneur, la composante FeedBack $U_{F B \_i j}(t)$ étant nulle (tracé en bleu Figure 11.f). Au contraire, lorsque le véhicule est en pleine charge, la composante FeedForward $U_{F F \_i j}(t)$ (tracé en rouge Figure 11.f) représente $70 \%$ de l'effort total $U_{a \_i j}(t)$ développé par chaque actionneur, la composante FeedBack $U_{F B \_i j}(t)$ assurant le complément (tracé en rouge Figure 11.f).

\section{Conclusion}

Face à une problématique aussi complexe que la coordination de l'ensemble des ADAS présents dans un véhicule, surtout dans un contexte autonome et connecté, une architecture hiérarchisée et générique est incontournable. Dans cette architecture, le Superviseur joue un rôle essentiel en raison, notamment, de la présence des modules Perception de l'environnement, Observation, Planification de trajectoire, Génération de mouvement et Diagnostic. Les variables de 


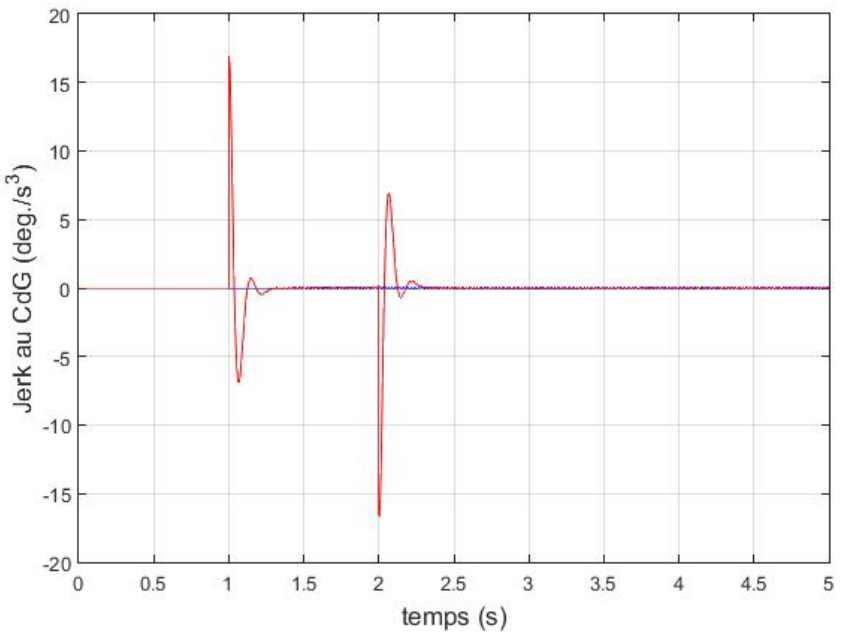

(a) Jerk au centre de gravité $J_{\varphi}(t)$

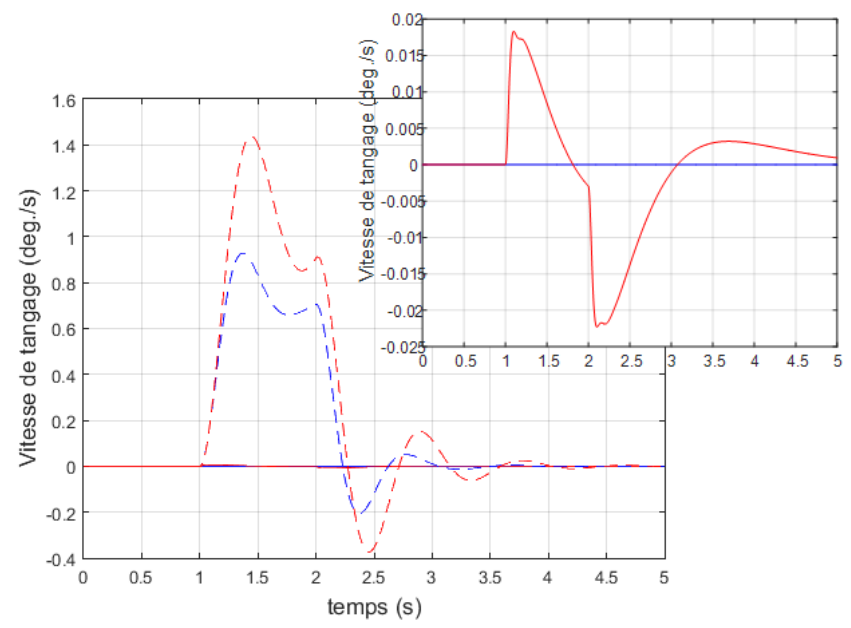

(c) Vitesse de tangage au centre de gravité $\Omega_{\varphi}(t)$
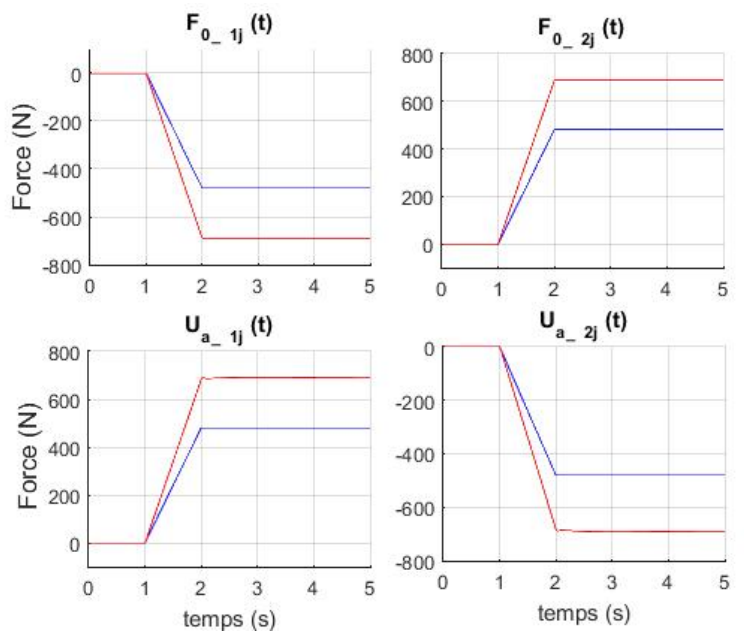

(e) Reports de charge $F_{0 \_i j}(t)$ et efforts des actionneurs $U_{a \_i j}(t)$

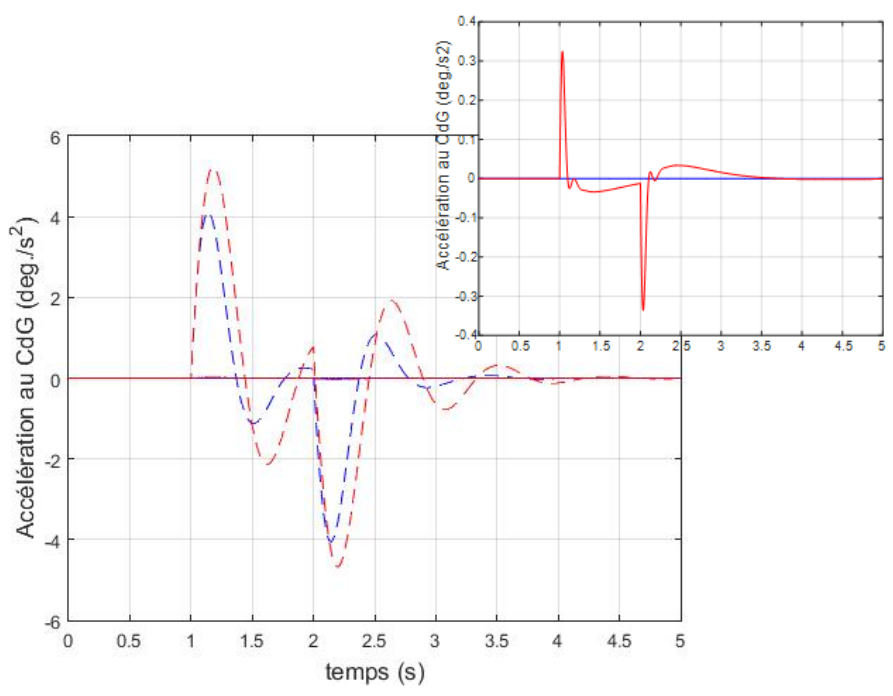

(b) Accélération de tangage au centre de gravité $A_{\varphi}(t)$

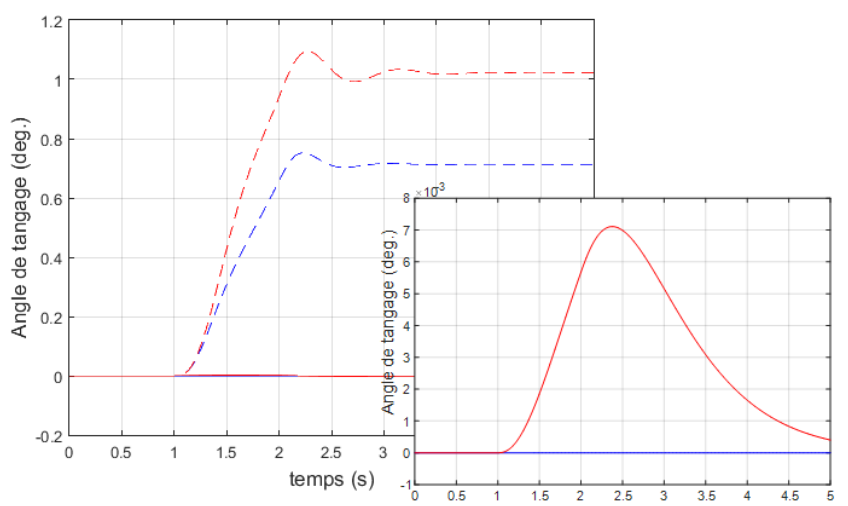

(d) Angle de tangage au centre de gravité $\varphi_{G}(t)$
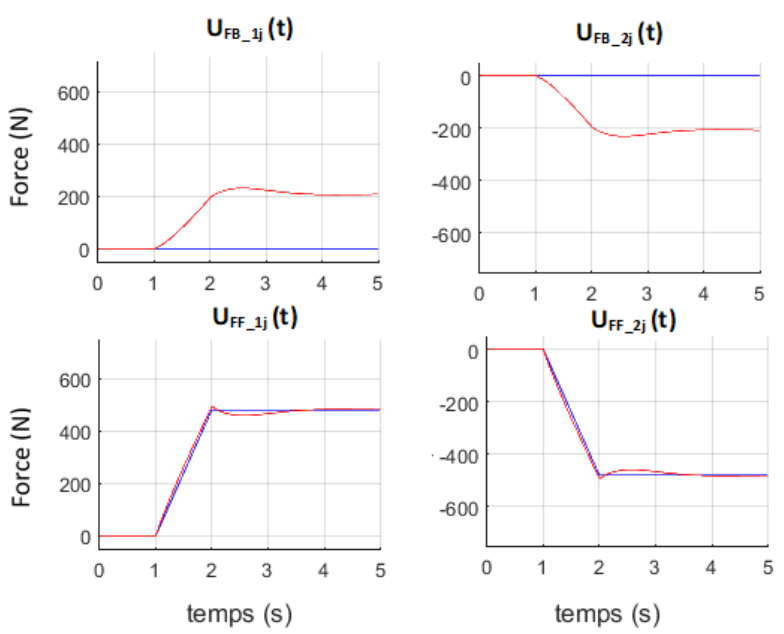

(f) Effort FeedBack résultant de la régulation $U_{F B \_i j}(t)$ et effort d'anticipation FeedForward $U_{F F \_i j}(t)$

Figure 11.: Performances temporelles 
références et les états paramétriques des régulateurs robustes transmis aux niveaux inférieurs dépendent du domaine de fonctionnement (confort, comportement routier ou sécurité) estimés par le superviseur. Ensuite, la Commande Globale située au niveau inférieur, à l'échelle fonctionnelle, permet de définir des stratégies générales indépendamment des contraintes liées aux solutions technologiques (mécanique, électrique, hydraulique,...). Puis, le niveau Répartition assure le passage de la Commande Globale à la Commande Locale, située à l'échelle organique, en choisissant la ou les liaisons au sol concernées par la mise en œuvre de la stratégie de commande. Enfin, le dernier niveau est composé de toutes les boucles de commandes locales indispensables pour atteindre les différents objectifs.

Pour des raisons didactiques, mais aussi de place disponible, l'exemple d'illustration proposé n'exploite volontairement pas tout le potentiel de cette architecture. En effet, le domaine d'étude de cet exemple se résume à une situation de freinage en ligne droite sur route sèche, lisse et horizontale. Ainsi, dans le domaine associé au confort, l'objectif est de tenir la caisse sous sollicitations conducteur par action sur la suspension. Les performances temporelles simulées mettent en évidence l'intérêt d'une telle approche pour atteindre l'objectif.

\section{Bibliographie}

VIVAS-López, C. A., HERÁNDEZ-ALCANTARA, D., TUDÓN-MARTNEZ, J. C., and MORALES-MENENDEZ, R. (2013). Review on Global Chassis Control. IFAC Proceedings Volumes, 46(2), 875-880.

PACEJKA, H. (2005). Tire and vehicle dynamics. Elsevier, Oxford.

GILLESPIE T.D. (1992). Fundamentals of vehicle dynamics. SAE International, Warrendale.

GORDON, T., HOWELL, M., and BRANDAO, F. (2003). Integrated control methodologies for road vehicles. Vehicle System Dynamics, 40(1-3) :157-190.

LANUSSE, P., MALTI, R. and MELCHIOR, P. (2013), CRONE control system design toolbox for the control engineering community : tutorial and case study, Philosophical Transactions of the Royal Society of London A : Mathematical, Physical and Engineering Sciences, The Royal Society, 371/1990, 20120149.

SENAME, OLIVIER and GASPAR, PETER and BOKOR, JÓZSEF (2013), Robust control and linear parameter varying approaches : application to vehicle dynamics, Springer, 437.

OUSTALOUP, A. (1991), La commande CRONE, Hermès Editor : Paris, France.

MOnOt, N., MOREAU, X., BEnINE-NETO, A., RIZZO, A., and AIOUN, F. (2017). Dynamic stability control system : the CRONE approach. 20th World Congress of the International Federation of Automatic Control, IFAC, 50(1), 13822-13827, Toulouse, France, 9-14 July.

JOHANSEN, T. A., and FOSSEN, T. I. (2013). Control allocation - A survey. Automatica, 49(5), 1087-1103.

BENINE-NETO, A., MOREAU, X., and LANUSSE, P. (2017). Robust control for electro-mechanical anti-lock braking system : the CRONE approach. 20th World Congress of the International Federation of Automatic Control, IFAC, 50(1), 12575-12581, Toulouse, France, 9-14 July.

BOUVIN, J.-L., MOREAU, X., BEnine-NETO, A., OUSTAlOUP, A., SERRIER, P., and HERnETTE, V. (2017). CRONE control of a pneumatic self-leveling suspension system. 20th World Congress of the International Federation of Automatic Control, IFAC, 50(1), 13816-13821, Toulouse, France, 9-14 July.

MORAND, A., MOREAU, X., MELCHIOR, P., MOZE, M., and GUILLEMARD, F. (2015). CRONE cruise control system. IEEE Transactions on Vehicular Technology, 65(1), 15-28.

ATTIA, R., ORJUELA, R., and BASSET, M. (2014). Dual-mode Control Allocation for Integrated Chassis Stabilization. IFAC Proceedings Volumes, 47(3), 11219-11224.

NOUILlANT, C., ASSADIAN, F., MOREAU, X., and OUSTALOUP, A. (2002). Feedforward and CRONE feedback control methods for automobile abs. Journal of Vehicle System Dynamics, ISSN : 1744-5159, 38(4), pp.293-315.

OUSTAlOUP, A., MELCHIOR, P., LANUSSE, P., COIS, O. and DANCLA, F. (2000), The CRONE toolbox for Matlab, Computer-Aided Control System Design, 2000. CACSD 2000. IEEE International Symposium on, IEEE, 190-195.

GAY F. (2000). Lois de commande en vue du Contrôle Global de châssis d'un Véhicule : le CGC. Thèse CIFRE PSA, Nantes. 
MORAND A. (2014) - Commande assistée au conducteur basée sur la conduite en formation de type "Banc de poissons". Thèse CIFRE PSA, Bordeaux.

LETEVE A. (2014) - Etude de l'influence des suspensions de véhicule de tourisme sur le confort vibratoire, le comportement routier et les limites de fonctionnement : l'approche CRONE en matière de formalisation, d'analyse et de synthèse. Thèse CIFRE PSA, Bordeaux.

SAE Inernational - On-Road Automated Vehicle Standards Committee, Taxonomy and Definitions for Terms Related to On-Road Motor Vehicle Automated Driving Systems. Rapprt d'information, Janvier 2014. 